\title{
EFECTOS DEL NIVEL DE ABSTRACCIÓN LÉXICA SOBRE LA MEMORIA A CORTO PLAZO
}

\author{
Armel Brizuela Rodríguez*
}

\begin{abstract}
RESUMEN
Se planteó un diseño experimental intrasujetos para determinar si el nivel de abstracción del vocabulario influye sobre el recuerdo a corto plazo de estas. Se utilizó una muestra de 34 estudiantes universitarios, a quienes se les presentaron dos listas de veinte palabras cada una para que posteriormente anotaran la mayor cantidad de términos que pudieran recordar, así como también cinco conjuntos de oraciones de las cuales debían recordar el último vocablo de cada una, esto con el fin de medir memoria de trabajo. Se encontró una diferencia media significativa entre el recuerdo de palabras concretas y abstractas, $t(33)=-5.319, p=.000$. Finalmente, se discuten algunos resultados que no concuerdan con la teoría de la codificación dual, en el tanto en que también se encontró una diferencia significativa entre palabras abstractas que no tenían un vínculo semántico entre sí y aquellas que sí lo tenían.

Palabras clave: codificación dual, efecto de concreción, recuerdo a corto plazo, memoria de trabajo, diseño intrasujetos.
\end{abstract}

\begin{abstract}
In the present study is proposed a within-subjects experimental design to determine if the vocabulary level of abstraction influences the short-term memory of words. A sample of 34 college students who were presented with two lists of twenty words each, for later write the highest number of words they could remember, as well as five sets of sentences. From this, they must to remember the last word of each, this in order to measure working memory. It was founded a significant mean difference between the memory of concrete and abstract words, $t(33)=5319, p=.000$. Finally, it is discussed some results that are inconsistent with the dual coding theory, because it was founded a significant difference between abstract words that had no semantic link between itself and those who do have.
\end{abstract}

Key Words: Dual coding, concreteness effect, short-term memory, working memory, within-subjects design.

\section{Introducción}

Una gran cantidad de investigaciones se han realizado desde la década de los 80 sobre el efecto de concreción, según el cual las palabras concretas tienen una mayor ventaja en el procesamiento cognitivo respecto de las abstractas. Desde las técnicas clásicas de papel y lápiz hasta las más modernas de imagenología funcional por resonancia magnética, en la investigación hecha sobre este tema siempre ha estado el interés por encontrar los mecanismos que expliquen este fenómeno. Por otro lado, en la actualidad existe el interés de la Psicometría por vincular sus intereses con los de las teorías cognitivas del procesamiento de la información. Tal interés se ha visto catalizado por la necesidad de aportar evidencias de validez de constructo

Instituto de Investigaciones Psicológicas. Universidad de Costa Rica.

Recepción: 27/06/12. Aceptación: 30/10/12. 
en las pruebas de evaluación psicológica y educativa. En este sentido, los investigadores han asumido la misión de aclarar con mayor precisión qué es exactamente lo que sus instrumentos miden, lo cual es fundamental en aras de realizar interpretaciones acertadas sobre los puntajes obtenidos en dichas pruebas.

Ante este panorama, la presente investigación sigue esta línea de investigación en la que, a partir de la teoría desarrollada por los psicólogos cognitivos durante muchos años, se pretende identificar qué características de los reactivos utilizados típicamente en pruebas psicológicas son relevantes para predecir la conducta cognitiva de los sujetos. Específicamente, en este proyecto interesa averiguar de qué manera el nivel de abstracción de las palabras incide sobre los procesos cognitivos de los sujetos. De esta manera, si se encontrara que el efecto de concreción efectivamente incide sobre el rendimiento mostrado por los sujetos, cabría la posibilidad de proponer nuevas investigaciones en las que se tomara en cuenta este factor para intentar predecir los tradicionales indicadores psicométricos empleados en la evaluación de la calidad de un ítem: índices de dificultad y de discriminación.

En lo que respecta a la investigación desarrollada en torno a este tema, podemos encontrar tres grandes ejes organizadores: 1) enfoque de procesamiento de la información en cuanto a la teoría de la codificación dual (Paivio, 1990), 2) explicaciones alternativas y matizaciones al modelo de Paivio y 3) neurociencia de la codificación semántica.

En lo que respecta al primer eje, encontramos estudios en los cuales se aportan argumentos a favor de la mayor facilidad con la que se procesan los términos concretos en comparación con los abstractos debido a la codificación dual que opera sobre aquellos. Por ejemplo, en estudios como los de Kroll y Merves (1986) y Walker y Hume (1999) se encuentra que en tareas de decisión léxica se clasifican más rápido las palabras concretas que las abstractas, así como también existe una mayor ventaja para el recuerdo hablado y escrito de palabras concretas, tanto en tareas de memoria retrospectiva como prospectiva.

En lo que respecta al segundo eje mencionado, existen investigaciones que cuestionan el poder explicativo de la propuesta de Paivio (1990), por lo que proponen que es el contexto en el cual se presentan las palabras lo que permite una diferencia en cuanto a la facilidad en el procesamiento de ciertas palabras (Schwanenflugel y Shoben, 1983). También se ha propuesto (Haberlandt y Graesser, 1985) que existe un efecto importante de las características de las palabras, tales como longitud o frecuencia de uso en su velocidad de procesamiento. Por su parte, Schwanenflugel y Stowe (1989) encontraron una mayor lentitud para evaluar el sentido de oraciones con palabras abstractas en contextos no significativos. No obstante, ambos efectos dejaron de observarse cuando las palabras se presentaron en contextos significativos. Otra variable relevante ha sido la edad (Markovits y Vachon, 1990): se ha visto que para sujetos entre los 13 y 16 años de edad, el razonamiento con contenidos abstractos es más difícil que con contenidos concretos. Por su parte, Sadoski, Goetz y Fritz (1995) han planteado un modelo causal en el cual la concreción afectaba en gran medida la comprensibilidad y el recuerdo, y que la concreción y la familiaridad influían en el interés. También se ha visto que los textos concretos son mejor recordados que los abstractos, aunque la magnitud de la ventaja varía en función del tipo de texto (Sadoski, Goetz y Rodríguez, 2000). Finalmente, en cuanto a este segundo eje de investigaciones, Sa'di y Yamini (2006) han encontrado que en los textos concretos, el contexto tiene un efecto negativo sobre el recuerdo, dado que los sujetos en su estudio se desempeñaron mejor en ausencia de un contexto significativo. Los investigadores especulan que este último resultado pudo darse debido a que los textos coherentes aportan una macroestructura que hace al individuo olvidarse fácilmente de 
captar los detalles del texto, lo cual no ocurre en los textos no significativos, en los que la ausencia de una estructura obliga al individuo a concentrarse en los detalles de cada oración.

En cuanto al tercer eje, se han propuesto una gran cantidad de zonas cerebrales relacionadas con el procesamiento de contenidos concretos y abstractos. En este sentido, los patrones diferenciales de activación neuronal relacionados con la abstracción o la concreción del vocabulario han aportado evidencia importante a favor de la teoría de que en el procesamiento semántico del vocabulario opera una codificación de dual (Holcomb, Kounios, Anderson y West, 1999; Griesbrecht, Camblin y Swaab, 2004; Huang, Lee y Federmeier, 2010). Así mismo, West y Holcomb (2000) han observado en el cerebro potenciales relacionados con el evento que indican una activación neural más generalizada, lo cual se ha considerado como evidencia a favor de la teoría de codificación dual. Nittono, Suehiro y Hori (2002) por su parte encontraron que las palabras más imaginables provocaron patrones de activación en el hemisferio derecho del cerebro, además de la clásica activación en el hemisferio izquierdo. Just, Newman, Keller, McEleney y Carpenter (2004) han propuesto que las oraciones en las que se requiere manipular imágenes mentales provocan la activación de regiones (especialmente el surco intraparietal) que a su vez inciden en zonas asociadas a tareas de visualización como la rotación mental. Por otro lado, la comprensión de información abstracta (que no requiere de una gran visualización de los conceptos) está acompañada por la activación de los lóbulos temporales izquierdos superior y medio, ambos asociados al procesamiento semántico y al conocimiento del mundo. Así mismo, Binder, Westbury, McKiernan, Possing y Medler (2005) han identificado áreas en el lóbulo temporal izquierdo que se activan ante la presencia de palabras concretas y abstractas, mientras que las regiones bilaterales -que incluyen la circunvolución angular y el córtex dorsal prefrontal- muestran una mayor actividad en el caso de las palabras concretas. También afirman que el léxico abstracto provoca una mayor actividad en las regiones inferiores frontales izquierdas, las cuales han sido asociadas a procesos fonológicos y verbales que operan en la memoria de trabajo. En esta misma línea, Sabsevitz, Medler, Seidenberg y Binder (2005) plantean que, en comparación con los sustantivos concretos, los abstractos producen una mayor activación bilateral en las áreas temporal (medial y ventral), parietal (posterior-inferior), dorsal prefrontal y en la corteza posterior cingulada, mientras que los sustantivos abstractos provocan una gran actividad en el hemisferio izquierdo (específicamente, las cortezas temporal superior y frontal inferior). Por su parte, Papagnoa, Fogliataa, Catricaláa y Miniussic (2009) identificaron una disminución en el rendimiento de tareas con palabras abstractas cuando se estimula el lóbulo temporal izquierdo, mientras que para el vocabulario concreto el rendimiento empeoró cuando se afectó el temporal derecho, a partir de lo cual plantean que el léxico abstracto se almacena en la región posterior del giro temporal superior izquierdo y posiblemente en el giro frontal inferior, mientras que las áreas involucradas en el almacenamiento de elementos concretos incluyen la corteza temporal derecha. Sin embargo, también existen investigaciones que contradicen los resultados anteriores, como la de Jessen, Heun, Erb, Granath, Klose, Papassotiropoulos y Grodd (2000), quienes, para las palabras concretas, han descubierto una mayor actividad en los lóbulos parietales inferiores (izquierdo y derecho), frontal inferior izquierdo y en el lóbulo parietal superior. A partir de este patrón de activación tan distribuido en el encéfalo, los investigadores afirman que ninguna de las teorías propuestas hasta el momento (la de codificación dual y la de disponibilidad del contexto) es capaz por sí sola de explicar el efecto de concreción.

En términos generales, la propuesta de Paivio consiste en que el sistema cognitivo se compone de dos subsistemas independientes: el verbal (encargado de procesar estímulos 
lingüísticos organizados secuencialmente) y el no verbal (encargado de procesar imágenes mentales que corresponden icónicamente a objetos naturales). En el primer sistema encontramos una especialización hacia estímulos abstractos, mientras que en el segundo se especializa en información abstracta. Sin embargo, Paivio ha propuesto que existe la posibilidad de que ambos subsistemas trabajen en conjunto sobre un estímulo particular, lo cual permite que se dé un procesamiento más eficaz de dicho estímulo.

Tomando en cuenta los resultados anteriores, la presente investigación se propone establecer cuál es el efecto del tipo de palabra (concreta vs. abstracta) sobre el recuerdo a corto plazo. En este sentido, se plantea que (1) habrá una diferencia en cuanto a la cantidad de palabras que recuerdan los sujetos en función de si estas son concretas o abstractas, (2) los sujetos recordarán más palabras concretas que abstractas sin importar si estas están relacionadas semánticamente o no y (3) independientemente de la capacidad de memoria de trabajo de los sujetos, estos recordarán más palabras concretas que abstractas. Para ello, se propone una metodología experimental que será descrita a continuación.

\section{Método}

\subsection{Participantes}

La muestra estuvo conformada por 34 estudiantes universitarios. Para el cálculo del tamaño de la muestra se utilizó el software Gpower. Específicamente, se escogió la familia de pruebas t para comparar la diferencia entre dos medias dependientes, es decir, pareadas. El cálculo se hizo tomando en cuenta un poder estadístico del $80 \%$, un nivel de confianza del $95 \%$ (a dos colas) y un tamaño del efecto medio (0.5), el cual se utiliza cuando no se tiene a disposición una investigación similar en diseño, tareas y tipos de variables incluidas en el estudio en cuestión.

\section{$2.2 \quad$ Materiales}

Los materiales utilizados fueron los siguientes:

(1) Lista de 20 palabras no relacionadas entre sí (10 abstractas y 10 concretas) extraídas del sitio web http://www.psy.uwa.edu. au/mrcdatabase/uwa_mrc.htm. En este sitio web, se solicitaron dos listados de palabras: (a) Palabras concretas (índices de concreción e imaginabilidad entre 600 y 700$)$ y (b) palabras abstractas (índices entre 200 y 300). Las palabras utilizadas fueron las siguientes: Términos abstractos: Incertidumbre (Uncertainty), excepción, (Exception), diferencia (Difference), influencia (Influence), intención (Intention), motivo (Motive), normal (Normal), preferencia (Preference), reforma (Reformation) y tendencia (Tendency); Términos concretos: cuarto (Bedroom), sangre (Blood), cigarro (Cigarrete), diamante (Diamond), limón (Lemon), pants (Pantalones), radio (Radio), Sol (Sun), ventana, (Window) y galleta (Cookie).

(2) Lista de 20 palabras (10 concretas y 10 abstractas) que cumplieran con el requisito anterior y que además se relacionaran semánticamente. Los términos abstractos se refirieron a la investigación científica mientras que los concretos, a aparatos electrónicos. Los vocablos utilizados fueron los siguientes: Términos abstractos sobre ciencia: experimento (Experiment), hipótesis (Hypothesis), ciencia (Science), idea (Idea), explicación (Explanation), análisis (Analysis), descubrimiento (Discovery), investigación (Research), conocimiento (Knowledge), descripción (Description); Términos concretos sobre tecnología: teléfono (Phone), televisor 
(Television), computadora (Computer), automóvil (Car), radio (Radio), submarino (Submarine), avión (Airplane), ventilador (Fan), microondas (Microwave oven), refrigeradora (Fridge).

(3) Presentación en PowerPoint para mostrar a los sujetos el material verbal de los puntos 1 y 2 .

(4) Folleto con espacio para escribir las palabras recordadas de las listas (puntos 1 y 2) y las respuestas a un test de memoria de trabajo.

\subsection{Diseño y estrategia de análisis}

Para la presente investigación se propuso un diseño intrasujetos en el que todos los sujetos recibieron el mismo estímulo. La creación de dos grupos es simplemente una medida de contrabalanceo, para controlar el efecto que podría tener la práctica en los sujetos, por cuanto hacer la primera tarea podría facilitar llevar a cabo la segunda. Una vez obtenidos los datos, se ejecutaron pruebas t para muestras dependientes y un ANCOVA.

\subsection{Procedimiento}

Se formaron dos grupos de sujetos, 17 personas en cada uno. A un grupo se le presentó primero la lista de palabras no relacionadas y se les pidió que anotaran las que recordaran, luego se les presentó la lista de vocablos relacionados y se les solicitó que escribieran todas las que recordaran. Cabe señalar que ambos listados fueron presentados durante un breve periodo (se daba un intervalo de tiempo de 0,5 segundos entre la aparición de una palabra y la aparición de otra) y a continuación se les solicitaba a los sujetos que llevaran a cabo las tareas mencionadas. En el otro grupo, se llevaron a cabo los mismos procedimientos, con la única diferencia de que primero se les enseñó la lista de términos relacionados y luego la de palabras no relacionadas. Finalmente, en ambos grupos se aplicó una prueba de memoria de trabajo.
Para ello, el investigador les leyó en voz alta a los sujetos 5 series de oraciones, cada una de las cuales estaba compuesta por dos, tres, cuatro, cinco y seis oraciones. La tarea de los participantes consistía en escuchar la lectura de cada serie y anotar, una vez finalizada esta, la última palabra de cada oración.

\section{Resultados}

Se utilizó la prueba de KolmogorovSmirnov para verificar que las variables utilizadas en los análisis fueran normales. En todos los casos, se observaron valores no significativos de la z de Kolmogorov-Smirnov, excepto la lista de términos abstractos no relacionados $(z=1.496$, $p=.023)$, por lo cual se procedió a ejecutar las pruebas t para muestras relacionadas, así como también el ANCOVA para verificar si la covariable Memoria de trabajo explica los resultados obtenidos. Es decir, se verificó si los resultados aportaban evidencia para las hipótesis sobre (1) diferencias entre el recuerdo de términos abstractos y concretos, (2) sobre igualdad en términos de si las palabras estaban relacionadas o no y (3) sobre la no irrelevancia de la capacidad en memoria de trabajo para explicar las diferencias encontradas.

En lo que respecta a las pruebas $t$ (cuyos resultados se muestran en la tabla 1), en el quinto par contrastado se observa una diferencia significativa entre el recuerdo de palabras abstractas y concretas, a favor de estas últimas, lo cual aporta evidencia a favor de la hipótesis de que se recuerdan una mayor cantidad de términos concretos que abstractos. Sin embargo, también el primer par es significativo, lo cual no concuerda con la teoría de la codificación dual, en virtud de la cual no es la disponibilidad del contexto semántico lo que favorece el recuerdo de palabras, sino solamente su nivel de abstracción. Es decir, en cuanto a la hipótesis de que los sujetos recordarían más vocablos concretos que abstractos sin importar la relación entre las palabras, no se puede afirmar que el vínculo 
semántico no influya sobre la cantidad de palabras recordadas cuando estas son abstractas. Por otro lado, el segundo par contrastado está en consonancia con la mencionada hipótesis, es decir, que la disponibilidad del contexto (en este caso, el hecho de contar con un contexto o campo semántico que agrupe a las palabras) no afecta el recuerdo.
Por otro lado, los datos presentados en la tabla 2 refuerzan el planteamiento sobre el efecto que podría tener el vínculo semántico sobre el recuerdo de palabras. Tal y como se observa, los pares contrastados con tamaños de efecto más pequeños son aquellos en los que se contempla la lista de palabras concretas relacionadas. En los demás pares, se observa un

TABLA 1

Prueba $t$ para muestras dependientes

\begin{tabular}{|c|c|c|c|c|c|c|c|}
\hline Par contrastado & DM & DE & gl & $t$ & \multicolumn{2}{|c|}{$95 \%$ IC } & $p$ \\
\hline Abstractas no relacionadas & 1.706 & 1.548 & 33 & -6.426 & 1.166 & 2.246 & .000 \\
\hline $\begin{array}{l}2^{\circ} \text { Concretas no relacionadas - } \\
\text { Concretas relacionadas }\end{array}$ & .294 & 1.733 & 33 & .990 & .899 & .310 & .329 \\
\hline $\begin{array}{l}3^{\circ} \text { Concretas no relacionadas - } \\
\text { Abstractas no relacionadas }\end{array}$ & 2.441 & 2.092 & 33 & -6.805 & 1.711 & 3.171 & .000 \\
\hline $\begin{array}{l}4^{\circ} \text { Concretas relacionadas - } \\
\text { Abstractas relacionadas }\end{array}$ & .441 & 1.988 & 33 & -1.294 & .252 & 1.135 & .205 \\
\hline $\begin{array}{l}5^{\circ} \text { Concretas }- \\
\text { Abstractas }\end{array}$ & 2.82 & 3.160 & 33 & -5.319 & 1.780 & 3.985 & .000 \\
\hline
\end{tabular}

TABLA 2

Medias, tamaños de efectos y poder estadístico para pares contrastados

\begin{tabular}{lllll}
\hline & Par contrastado & DM & d & $\mathbf{1 - \beta}$ \\
\hline $1 \quad \begin{array}{l}\text { Abstractas relacionadas } \\
\text { Abstractas no relacionadas }\end{array}$ & 1.706 & 1.10 & .99 \\
2 & $\begin{array}{l}\text { Concretas no relacionadas } \\
\text { Concretas relacionadas }\end{array}$ & .294 & 0.16 & .16 \\
& & & \\
3 & $\begin{array}{l}\text { Concretas no relacionadas } \\
\text { Abstractas no relacionadas }\end{array}$ & 2.441 & 1.16 & .99 \\
4 & $\begin{array}{l}\text { Concretas relacionadas } \\
\text { Abstractas relacionadas }\end{array}$ & .441 & 0.22 & .24 \\
& $\begin{array}{l}\text { Concretas } \\
\text { Abstractas }\end{array}$ & 2.882 & 0.89 & .99 \\
\hline
\end{tabular}


alto poder estadístico alcanzado, lo cual indica que si la presente investigación se replicara en las mismas condiciones y con instrumentos similares, existe una alta probabilidad de que se encuentre resultados muy parecidos a los del presente estudio. Así pues, el poder estadístico tan bajo alcanzado en los pares contrastados 2 y 4 señala la posibilidad de que, en otra muestra y con los instrumentos empleados en este estudio, podrían encontrarse resultados completamente diferentes.

En cuanto al efecto de la covariable Memoria de trabajo sobre el recuerdo de las palabras, en la tabla 4 se pueden ver los resultados obtenidos. En primer lugar, se realizó la prueba de homogeneidad de las pendientes de regresión $[F(4,128)=1.109, p=.355]$, en la cual se verificó que no existiera una interacción significativa entre el factor de agrupación (tipo de lista de palabras) y la covariable. Cabe señalar que para poder incorporar la covariable en el análisis estadístico de los datos, se trabajó con la cantidad de palabras recordadas por los sujetos en los cuatro tipos de listas. Para cumplir con el requisito esencial del ANCOVA de independencia de los errores, se le restó la media $(M=4.60)$ al conjunto de palabras recordadas por los sujetos a través de los cuatro tipos de las listas, así como también se sustrajo el promedio $(M=18.03)$ de los puntajes obtenidos en el test de memoria de trabajo. Este procedimiento permite ejecutar el ANOVA sobre los valores obtenidos, sin el efecto de lo que comparten (esto es, la media).

En la tabla 3, se presentan los resultados del ANCOVA, a partir de los cuales se puede concluir que la covariable como tal no resultó de ser de utilidad para controlar el efecto de la memoria de trabajo en la variable dependiente, en tanto no tuvo un efecto significativo sobre el recuerdo de palabras.

\section{Discusión}

En cuanto a los resultados obtenidos en el presente estudio, llaman la atención el tercer y cuarto par contrastados (ver tabla 1). En cuanto al tercero (al igual que con el segundo), se puede ver como evidencia a favor de la hipótesis de la codificación dual, sin embargo, era esperable que el cuarto par contrastado también fuera significativo. Este resultado inesperado podría deberse a que los sujetos utilicen estrategias diferentes para recordar palabras en función de si están relacionadas o no, lo cual tiene similitudes con los hallazgos de Sa'di y Yamini (2006), quienes encontraron que para el vocabulario concreto el recuerdo disminuía en presencia de un contexto significativo. Tal y como se aprecia en la tabla 1, en promedio, los participantes recordaron más vocabulario concreto no relacionado que la contraparte de léxico relacionado, mientras que con las abstractas ocurrió lo contrario. Siguiendo a, Sa'di y Yamini quizá la ausencia de contexto obligue a los sujetos a concentrarse en cada una de las palabras por separado, mientras que la presencia de un contexto provoque en los sujetos cierto descuido, quienes confiarían en que podrán recordar posteriormente los términos valiéndose de información contextual.

TABLA 3. ANCOVA

\begin{tabular}{llllll}
\hline Origen & Suma de cuadrados & gl & Media cuadrática & $\mathbf{F}$ & $\mathbf{p}$ \\
\hline $\begin{array}{l}\text { Memoria de } \\
\text { trabajo }\end{array}$ & .008 & 1 & .008 & .009 & .926 \\
\hline Tipo de lista & 9.239 & 3 & 3.080 & 3.199 & $\mathbf{. 0 2 6}$ \\
\hline Error & 126.102 & 131 & .963 & & \\
\hline Total & 398.560 & 136 & & & \\
\hline Total Corregida & 135.349 & 135 & & & \\
\hline
\end{tabular}


Otro aspecto interesante es la diferencia en cuanto a significancia que se dio entre el primer y segundo pares contrastados. Por un lado, los términos abstractos relacionados y no relacionados difirieron, mientras que esto no ocurrió en el caso de los términos concretos. Llama poderosamente la atención el hecho de que por un lado se recordaran más palabras abstractas relacionadas que concretas relacionadas. Además de la posible explicación expuesta en el párrafo anterior a este hallazgo, siguiendo a Martin y Chao (2001) y a Moore y Price (1999), también podría aventurarse la hipótesis de que no solo el nivel de abstracción del léxico influye en el recuerdo a corto plazo, sino que también, en el caso de las palabras concretas, se han observado diferencias en el procesamiento cognitivo. En este sentido, al parecer el cerebro trata de manera diferente el estímulo si este es una herramienta (u otro tipo de objetos artificiales) o una fruta (u otro tipo de cosas orgánicas). En la lista de términos concretos se incorporaron palabras que remitían a todo tipo de objetos, lo cual pudo haber generado un efecto de confundido que no fue apropiadamente controlado. Por tal razón, es importante que en futuras investigaciones se tomen en cuenta tanto las estrategias que utilizan los sujetos para memorizar palabras en función de si estas son concretas o abstractas, y de controlar el tipo de términos concretos que se utilicen como estímulos. En todo caso, estas recomendaciones implican que el modelo de Paivio no es capaz por sí solo de explicar las diferencias encontradas en el recuerdo a corto plazo de léxico.

Cabe señalar que el hecho de que el vínculo semántico en algunos tipos de lista resultara ser significativo sugiere que otras variables deberían estudiarse más como posibles candidatas para explicar la mayor facilidad con la que los sujetos recuerdan un tipo de palabras y no otro. En este sentido, para subsanar esta limitación de la investigación, se recomienda tomar en cuenta si los participantes implementan estrategias nemotécnicas diferentes para enfrentarse a tareas de recuerdo libre.
Finalmente, con respecto al interés planteado al inicio sobre averiguar si el nivel de abstracción de las palabras podría ser un predictor útil en la dificultad de ítems de comprensión de lectura, en la presente investigación se presentan algunas evidencias de que el vocabulario abstracto dificulta el procesamiento cognitivo de la información verbal. Por tal motivo, los resultados obtenidos servirán para una futura investigación en la que se pondrá a prueba si el léxico abstracto resulta ser de utilidad a la hora de predecir los índices de dificultad de los ítems verbales de la Prueba de Aptitud Académica de la Universidad de Costa Rica.

\section{Bibliografía}

Binder, J., Westbury, C., McKiernan, K., Possing, E. y Medler, D. 2005. Distinct Brain Systems for Processing Concrete and Abstract Concepts. Journal of Cognitive Neuroscience, 17(6), 1-13.

Del Río, D.y López-Higes, R. 2006. Efectos de la memoria operativa y de una carga de procesamiento en la comprensión de oraciones. Psicológica, 27, 79-95.

Fahandezh, F. y Yamini, M. 2006. Comprehension and Recall of Concrete and Abstract Texts with Reference to Dual-Coding and Context-Availability. Journal of Social Sciences \& Humanities of Shiraz University, 23(1), 21-29.

Giesbrecht, B., Camblin, C. y Swaab, T. 2004. Separable Effects of Semantic Priming and Imageability on World Processing in Human Cortex. Cerebral Cortex, 14(5), 521-529.

Gutiérrez, F., García, J., Carriedo, N., Vila, J. y Luzón, J. 2005. Dos pruebas de amplitud de memoria operativa para el razonamiento. Cognitiva, 17 (2), 183-210. 
Haberlandt, K. y Graesser, A. 1985. Component Processes in Text Comprehension and Some of Their Interactions. Journal of Experimental Psychology: General, 114 (3), 357-374.

Hiroshi, N.; Maki, S. y Tadao, H. 2002. Word imageability and N400 in an incidental memory paradigm. International Journal of Psychophysiology, 44, 219-229.

Holcomb, P., Anderson, J., Kounios, J. y West, W. 1999. Dual-Coding, Context-Availability, and Concretness Effects in Sentence Comprehension: An Electrophysiological Investigation. Journal of Experimental Psychology: Learning, Memory, and Cognition, 25 (3), 721-742.

Huang, H., Lee, C. y Federmeier, K. 2010. Imagine that! ERPs provide evidence for distinct hemispheric contributions to the processing of concrete and abstract concepts. NeuroImage, 49, 1116-1123.

Jessen, F., Heun, R., Erb, M., Granath, D., Klose, U., Papassotiropoulos, A. y Grodd, W. 2000. The Concreteness Effect: Evidence for Dual Coding and Context Availability. Brain and Language, 74 (1), 103-112.

Just, M., Newman, S., Keller, T., McEleney, A. y Carpenter, P. 2004. Imagery in sentence comprehension: an fMRI study. NeuroImage, 21, 112-124.

Kintsch, W. 1998. Comprehension / A Paradigm for Cognition. NY: Cambridge University Press.

Kroll, J. y Merves, J. 1986. Lexical Access for Concrete and Abstract Words. Journal of Experimental Psychology: Learning, Memory, and Cognition, 12 (1), 92-107.
Markovits, H. y Vachon, R. 1989. Conditional Reasoning, Representation, and Level of Abstraction. Developmental Psychology, 26 (6), 942-951.

Marvin, A. y Chao, L. 2001. Semantic memory and the brain: structure and processes. Current opinion in neurobiology, 11 (2), 194-201.

Moore, C. y Price, C. 1999. A functional neuroimaging study of the variables that generate category-specific object processing differences. Brain, 122, 943-962.

Nittono, H., Suehiro, M. y Hori, T. 2002. Word imageability and N400 in an incidental memory paradigm. International Journal of Psychophysiology, 44, 219-229.

Paivio, A. 1990. Mental Representations / A Dual Coding Approach. NY: Oxford University Press.

Papagno, C., Fogliata, A., Catricalà, E. y Miniussi, C. 2009. The lexical processing of abstract and concrete nouns. Brain Research, 1263 78-86.

Sabsevitz, D., Medler, D., Seidenberg, M. $\mathrm{y}$ Binder, J. 2005. Modulation of the semantic system by word imageability. NeuroImage, 27, 188 - 200.

Sadoski, M., Goetz, E. y Fritz, J. 1993. A Causal Model of Sentence Recall: Effects of Familiarity, Concreteness, Comprehensibility, and Interestingness. Journal of Literacy Research, 25 (1), 5-16.

Sadoski, M., Goetz, E. y Rodríguez, M. 2000. Engaging Texts: Effects of Concreteness on Comprehensibility, Interest, and Recall in Four Text Types. Journal of Educational Psychology, 92 (1), 85-95. 
Schwanenflugel, P. y Shoben, E. 1983. Differential Context Effects in the Comprehension of Abstract and Concrete Verbal Materials. Journal of Experimental Psychology: Learning, Memory, and Cognition, 9 (1), 82-102.

Schwanenflugel, P. y Stowe, R. 1989. Context Availability and the Processing of Abstract and Concrete Words in Sentences. Reading Research Quarterly, 24 (1), 114-126.

Smith, E. y Kosslyn, S. 2008. Procesos cognitivos / Modelos y bases neurales. Madrid: Pearson Educación.
Sternberg, R. 2011. Psicología cognoscitiva. México: Cengage Learning International.

Walker, I. y Hulme, C. 1999. Concrete Words Are Easier to Recall Than Abstract Words: Evidence for a Semantic Contribution to Short-Term Serial Recall. Journal of Experimental Psychology: Learning, Memory, and Cognition, 25 (5), 1256-1271.

West, W. y Holcomb, P. 2000. Imaginal, Semantic, and Surface-Level Processing of Concrete and Abstract Words: An Electrophysiological Investigation. Journal of Cognitive Neuroscience, 12 (6), 1024-1037. 